\section{Interactions négatives \\ entre récepteurs \\ nucléaires \\ et facteurs \\ transcriptionnels}

Virginie Vlaeminck-Guillem, Vincent Laudet, Martine Duterque-Coquillaud

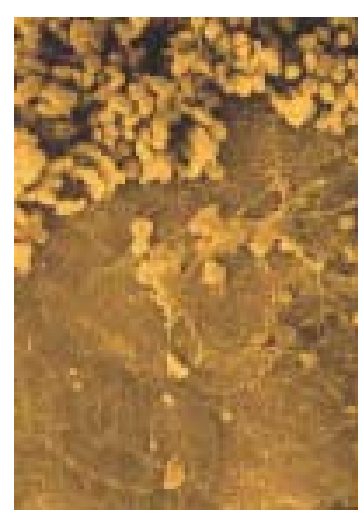

V. Vlaeminck-Guillem:

Clinique Marc Linquette, USNA, CHRU de Lille,

6, rue du Professeur Laguesse, 59037 Lille Cedex, France. V. Laudet: Cnrs UMR 5665, École Normale Supérieure de Lyon, 46, allée d'Italie, 69394 Lyon Cedex 07, France. M. Duterque-Coquillaud: Cnrs UMR 8526, Institut de Biologie de Lille, La cellule vivante BP 447, 1, rue Calmette, reçoit en permanence 59021 Lille Cedex, France. de nombreux signaux virginie.vlaeminck@wanadoo.fr extérieurs, parfois contradictoires, auxquels elle doit répondre de façon adaptée. Cette réponse requiert l'intégration de plusieurs signaux simultanés qui sont captés par deux types de récepteurs, les récepteurs membranaires et les récepteurs nucléaires. Les récepteurs membranaires, après interaction avec leur ligand, transmettent un signal d'activation vers des facteurs transcriptionnels nucléaires via une cascade de phosphorylations. Les récepteurs nucléaires, situés par définition dans le noyau, se comportent euxmêmes comme des facteurs transcriptionnels dont l'activité dépend de la liaison d'un ligand lipophile. Grâce à une organisation modulaire, comprenant un domaine de liaison à l'ADN, un domaine de liaison au ligand et un ou plusieurs domaines activateurs de la transcription, les récepteurs nucléaires sont capables de se fixer directement, le plus souvent sous forme 
dimérique, sur des séquences spécifiques situées dans les régions régulatrices des gènes cibles qu'ils activent en présence du ligand. Les ligands des récepteurs nucléaires comprennent notamment les stéroïdes, les hormones thyroïdiennes, la vitamine $D$ et l'acide rétinoïque. Ces ligands correspondent aux produits finaux de multiples voies métaboliques qui utilisent comme substrat principal un ou plusieurs nutriments issus de l'alimentation (tyrosine et iode pour les hormones thyroïdiennes, cholestérol pour les hormones stéroïdes, vitamine A pour l'acide rétinoïque...). Leurs actions physiologiques sont multiples mais, schématiquement, concourent plutôt à la différenciation cellulaire. Au contraire, les signaux véhiculés par les récepteurs membranaires induisent plutôt une prolifération cellulaire. L'orientation de la cellule vers la différenciation ou la prolifération résulte par conséquent de la prédominance relative de l'une ou l'autre de ces deux grandes voies de signalisation. Au niveau moléculaire, l'intégration de ces signaux contradictoires repose en partie sur des interactions négatives. Le but de cet article est d'illustrer les mécanismes d'interaction négative entre les récepteurs nucléaires et les autres facteurs transcriptionnels à travers deux grands processus physiopathologiques: l'inflammation et la cancérogenèse.

\section{Le contrôle de l'inflammation}

\section{NF-KB/Rel et récepteurs \\ des glucocorticoïdes}

La réponse inflammatoire est un processus complexe et crucial qui doit être à la fois efficace, pour permettre à l'organisme de résister aux agressions extérieures, et mesuré, pour éviter l'apparition de lésions cellulaires ou tissulaires. Cet équilibre délicat est assuré par l'action de facteurs pro- et anti-inflammatoires parmi lesquels on trouve le récepteur des glucocorticoïdes et les facteurs transcriptionnels membres de la famille NF-KB/Rel.

La famille $N F-\kappa B / R e l$, dont la protéine p65/RelA est le prototype, est constituée de facteurs transcriptionnels dont l'expression, ubiquitaire, est particulièrement élevée dans les cellules immunocompétentes. Ils stimulent l'expression de nombreux gènes codant pour des acteurs des réponses inflammatoire et immunitaire comme les cytokines ou des molécules d'adhérence cellulaire. Initialement cyto- plasmiques, les facteurs NF-KB (nuclear factor $\kappa B$ ) sont des hétérodimères inactifs dont l'activation, induite par l'action de signaux extracellulaires, dépend de deux événements protéolytiques: la maturation de la sous-unité p105 en une protéine $\mathrm{p} 50$ et la dégradation de $\mathrm{IKB}$ (inhibitor of $N F-\kappa B$ ), inhibiteur spécifique associé à NF-KB (Figure 1). Les facteurs NFKB sont alors transférés vers le noyau où ils exercent leur activité transcriptionnelle ciblée.

En l'absence de ligand, le récepteur des glucocorticoïdes (GR, glucocorticoid receptor) est séquestré dans le cytoplasme et maintenu sous forme inactive par association aux protéines de choc thermique (heat shock proteins) HSP90 et HSP56. La liaison du ligand induit un changement conformationnel du GR qui est de ce fait relargué des protéines HSP, phosphorylé et déplacé vers le noyau. II se fixe alors sous forme d'homodimère sur des séquences spécifiques localisées dans les régions régulatrices des gènes cibles. De nombreux gènes cibles dont l'expression est réprimée par le $G R$ sont au contraire activés par les facteurs NF-KB. In vitro, GR et NF-KB exercent un véritable antagonisme réciproque, chacun étant capable d'inhiber l'activité transcriptionnelle de l'autre [1].

Plusieurs hypothèses ont été formulées pour expliquer le mécanisme de cet antagonisme réciproque, qui ne résulte pas d'une diminution mutuelle de l'expression

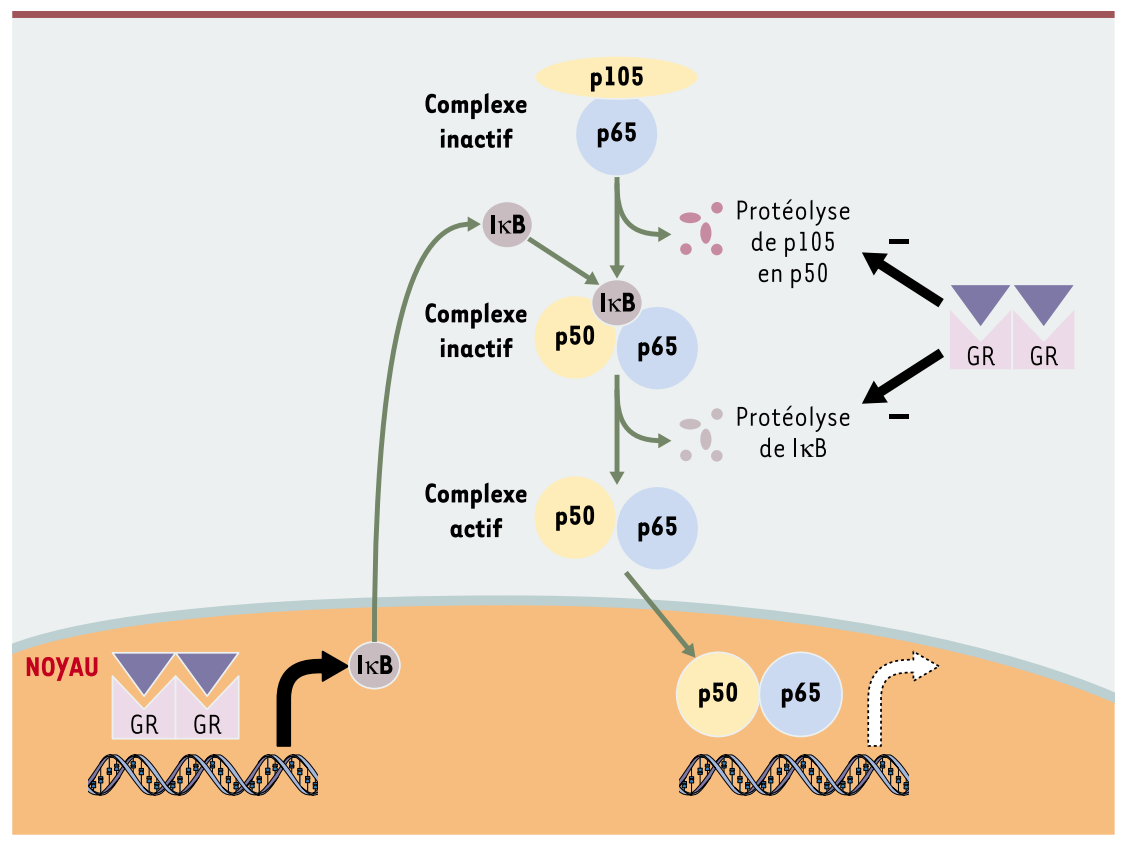

Figure 1. Inhibition de l'activité transcriptionnelle du facteur NFKB par le récepteur des glucocorticoïdes. L'activation du facteur NF-KB résulte d'une maturation progressive par protéolyse: (1) de la protéine p105 en sous-unité p50, et (2) de la protéine inhibitrice $\mid \kappa B$. La forme active de NF-KB est transférée dans le noyau où elle exerce son activité transcriptionnelle. Cette activité peut être inhibée par le récepteur des glucocorticoïdes (GR), qui, associé à son ligand, stimule l'expression de la protéine inhibitrice $\mid \kappa B[3,4]$ et inhibe sa protéolyse et celle de la pl05 [5]. 
intracellulaire de ces deux facteurs [2]. Il pourrait, par exemple, être dû à un effet sur la disponibilité des formes actives. Effectivement, l'expression de la protéine $I \kappa B \alpha$, sous-unité inhibitrice qui maintient NF- $\kappa B$ sous forme cytoplasmique, est stimulée par le GR [3, 4]. Le GR pourrait donc réprimer l'activité de p65/RelA par augmentation de sa séquestration intracytoplasmique (Figure 1). Le GR pourrait également inhiber directement le processus protéolytique aboutissant à la dégradation des inhibiteurs $I K B$ et à la maturation des précurseurs (Figure 1) [5]. Ces mécanismes sont cependant controversés: ils ne sont pas toujours reproductibles et semblent restreints à certains tissus ou types cellulaires $[6,7]$. Une autre hypothèse propose que l'inhibition réciproque de GR et NF-KB résulterait d'une interaction physique entre ces deux facteurs via leurs domaines de liaison à I'ADN $[1,8]$ : le domaine C du GR et le domaine Rel de NF-KB [9]. Cependant, l'interaction physique entre GR et NF- $K B$ ne semble pas interférer avec leur liaison à I'ADN [10] comme le fait par exemple l'interaction entre le récepteur nucléaire PPAR (peroxisome proliferator-activated receptor) et le facteur NRF2 sur le promoteur du gène de la thromboxane-synthétase de rat [11]. Une troisième hypothèse, en faveur actuellement, propose une compétition de GR et $N F-\kappa B$ pour un cofacteur commun. En effet, l'activité de ces facteurs de transcription est dépendante de leur interaction avec de nombreux cofacteurs nucléaires. Si un cofacteur commun à ces deux voies d'activation est présent dans le noyau en quantité limitante, les deux facteurs transcriptionnels peuvent entrer en compétition pour sa liaison: le plus abondant détournerait ce cofacteur à son profit et inhiberait l'activité transcriptionnelle du moins abondant. Ce mécanisme est appelé squelching par les auteurs anglosaxons (Figure 2). Parmi les cofacteurs utilisés à la fois par les récepteurs nucléaires et les facteurs NF-KB figurent des coactivateurs comme SRC-1 (steroid receptor coactivator -1 ). Ce sont des protéines qui assurent la liaison entre le facteur transcriptionnel et des complexes multiprotéiques activateurs qui, par acétylation des histones, induisent un relâchement de la chromatine permettant la transcription des gènes cibles. Une compétition pour la protéine SRC-1 expliquerait au moins partiellement l'interaction négative réciproque entre GR et NF- $K B$ [12]. D'autres candidats possibles sont les cofacteurs apparentés CBP et $\mathrm{p} 300$, dont la présence est nécessaire à un si grand nombre de facteurs transcriptionnels qu'ils sont actuellement définis comme des «cointégrateurs», c'est-à-dire des protéines dont la présence en quantité limitante favorise l'activité d'un facteur transcriptionnel prédominant. Une compétition pour la protéine CBP a été évoquée pour expliquer de nombreuses interactions négatives entre facteurs transcriptionnels, comme par exemple l'antagonisme entre les récepteurs 
nucléaires et le complexe AP-1 (activating protein-1) [13]. Elle ne semble pas devoir être retenue pour l'interaction entre GR et NF-KB, même si ces deux facteurs utilisent CBP comme cofacteur [14]. Finalement, un autre mécanisme récemment décrit pourrait concourir à l'antagonisme entre GR et NF- $K B$. NF- $K B$ est capable de stimuler l'expression de l'isoforme $\beta$ du GR (GR $\beta$ ), isoforme qui se comporte comme un inhibiteur endogène naturel de l'isoforme fonctionnelle $\alpha$ du GR [15]. NF$\kappa B$ pourrait donc inhiber l'activité du $G R$ en modifiant l'équilibre entre les isoformes $\alpha$ et $\beta$.

\section{Autres interactions négatives avec NF- KB}

Le GR n'est pas le seul récepteur nucléaire impliqué dans des interactions négatives avec NF-KB. Le récepteur des androgènes exerce lui aussi un antagonisme transcriptionnel réciproque avec NF-KB même si la signification physiologique de cette interaction n'est pas aussi claire que pour le GR [2]. Dans ce cas, la transcription du gène codant pour le récepteur des androgènes pourrait être inhibée directement par la fixation de NF-KB sur son promoteur [16]. Des interactions entre $N F-\kappa B$ et le récepteur des œstrogènes ou de la progestérone ont également été décrites [17-19] et pourraient avoir des conséquences physiologiques majeures, comme par exemple l'immunosuppression relative lors de la grossesse. Plus étayée est l'action anti-inflammatoire qu'exercent les récepteurs PPAR par leur interaction avec NF-KB [20, 21]. Les PPAR jouent un rôle majeur dans le métabolisme lipidique et glucidique. Alors que PPAR $\gamma$ favorise le stockage des lipides en stimulant la différenciation adipocytaire, PPAR $\alpha$ stimule la dégradation oxydative des acides gras. Les ligands des PPAR sont des molécules naturelles, dérivées du métabolisme des acides gras, ou des molécules synthétiques, les fibrates, qui sont utilisées en thérapie comme agents hypolipémiants. Les études cliniques ont souligné l'intérêt des fibrates dans la prévention de l'infarctus du myocarde en montrant leur effet inhibiteur sur la progression de l'athérome artériel. Cet effet antiathéromateux semble résulter directement de l'activité anti-inflammatoire de PPAR $\alpha$ [20]. PPAR $\alpha$, stimulé par les fibrates, inhibe en effet l'activité transcriptionnelle de NF-KB sur le promoteur de l'interleukine-6, une cytokine pro-inflammatoire sécrétée dans la paroi artérielle, et de la cyclo-oxygénase 2 , une enzyme responsable de la production des prostaglandines et des leucotriènes $[20,21]$. Si un mécanisme de compétition pour un cofacteur commun semble là encore probable, le cofacteur consommé n'est pas la protéine CBP dont la surexpression ne permet pas de lever l'inhibition transcriptionnelle de $N F-\kappa B[21]$.

\section{Le contrôle anti-oncogénique}

Nombre de facteurs transcriptionnels codés par des oncogènes, comme les membres de la famille ETS ( $\varepsilon$ twenty-six transcription factor) et le complexe AP-1, sont le substrat final d'une cascade de phosphorylations activée par un récepteur membranaire. L'activation pathologique de ces oncogènes peut conduire au développement de tumeurs par dérèglement de la prolifération cellulaire. À l'inverse, les récepteurs nucléaires sont souvent de puissants inducteurs de la différenciation cellulaire. Les récepteurs de l'acide rétinoïque, RAR (retinoic acid receptor) et RXR, en sont un exemple particulièrement démonstratif puisque leur expression et leur activation apparaissent nécessaires au maintien d'une différenciation épithéliale dans de nombreuses muqueuses digestives ou respiratoires [22]. L'acide rétinoïque est d'ailleurs utilisé pour induire la différenciation cellulaire dans certaines formes de leucémie comme la leucémies aiguë promyélocytaire qui résulte habituellement d'une translocation du gène codant pour le récepteur RAR et pour laquelle l'acide rétinoïque induit la différenciation des promyélocytes cancéreux [22]. Les effets des récepteurs nucléaires et des autres facteurs transcriptionnels apparaissent donc globalement antagonistes in vivo, comme l'illustrent bien les effets de Flil, membre de la famille ETS, et de RAR $\alpha$, récepteur de l'acide rétinoïque, dans des cellules souches hématopoïétiques en culture. En effet, la différenciation érythroïde normalement induite par RAR $\alpha$ sur le promoteur du gène codant pour le récepteur RARß2 est bloquée par Flil [23], qui détourne ces cellules vers la prolifération. Cet effet de Flil peut aller jusqu'à la transformation cellulaire, comme le montre le développement d'érythroleucémies murines [24] associé à la surexpression de Flil secondaire à des intégrations rétrovirales chez la souris.

Les interactions entre les récepteurs nucléaires et le complexe AP-1 ont été bien étudiées pour les promoteurs des gènes de la proliférine murine et de l'ostéocalcine humaine $[25,26]$. L'activité de ces promoteurs est stimulée par l'exposition cellulaire aux esters de phorbol (dont l'action est relayée par le complexe AP-1), alors qu'elle est inhibée par l'administration de glucocorticoïdes (pour la proliférine) ou d'acide rétinoïque (pour l'ostéocalcine). L'organisation de ces promoteurs est de type «composite», c'est-à-dire formée d'une juxtaposition de sites reconnus par AP-l et de sites reconnus par GR $[25,26]$. L'antagonisme fonctionnel 
entre GR et AP-1 peut cependant se produire en l'absence de site composite comme l'a montré l'étude du promoteur du gène de la collagénase humaine qui ne contient pas d'élément de réponse pour GR mais un unique site $A P-1$, nécessaire à l'activité répressive de GR sans pour autant que le GR s'y fixe [27-29]. Des résultats similaires ont été rapportés avec d'autres récepteurs nucléaires comme les récepteurs de l'acide rétinoïque [30], des hormones thyroïdiennes [31] ou des stéroïdes sexuels [32]. La répression qu'exerce le $\mathrm{GR}$, et les récepteurs nucléaires en général, sur certains promoteurs ne nécessite donc pas la liaison à l'ADN, ni même un domaine de liaison à I'ADN intact.

Quel est le mécanisme de l'antagonisme entre AP-1 et les récepteurs nucléaires? Les récepteurs nucléaires et les membres du complexe AP- 1 interagissent entre eux de façon directe via leurs domaines respectifs de liaison à I'ADN : domaine $C$ des récepteurs nucléaires et le domaine leucine-zipper des facteurs AP-1 [29, 3234]. Ainsi, le domaine de liaison à l'ADN des facteurs transcriptionnels ne constitue pas seulement une zone

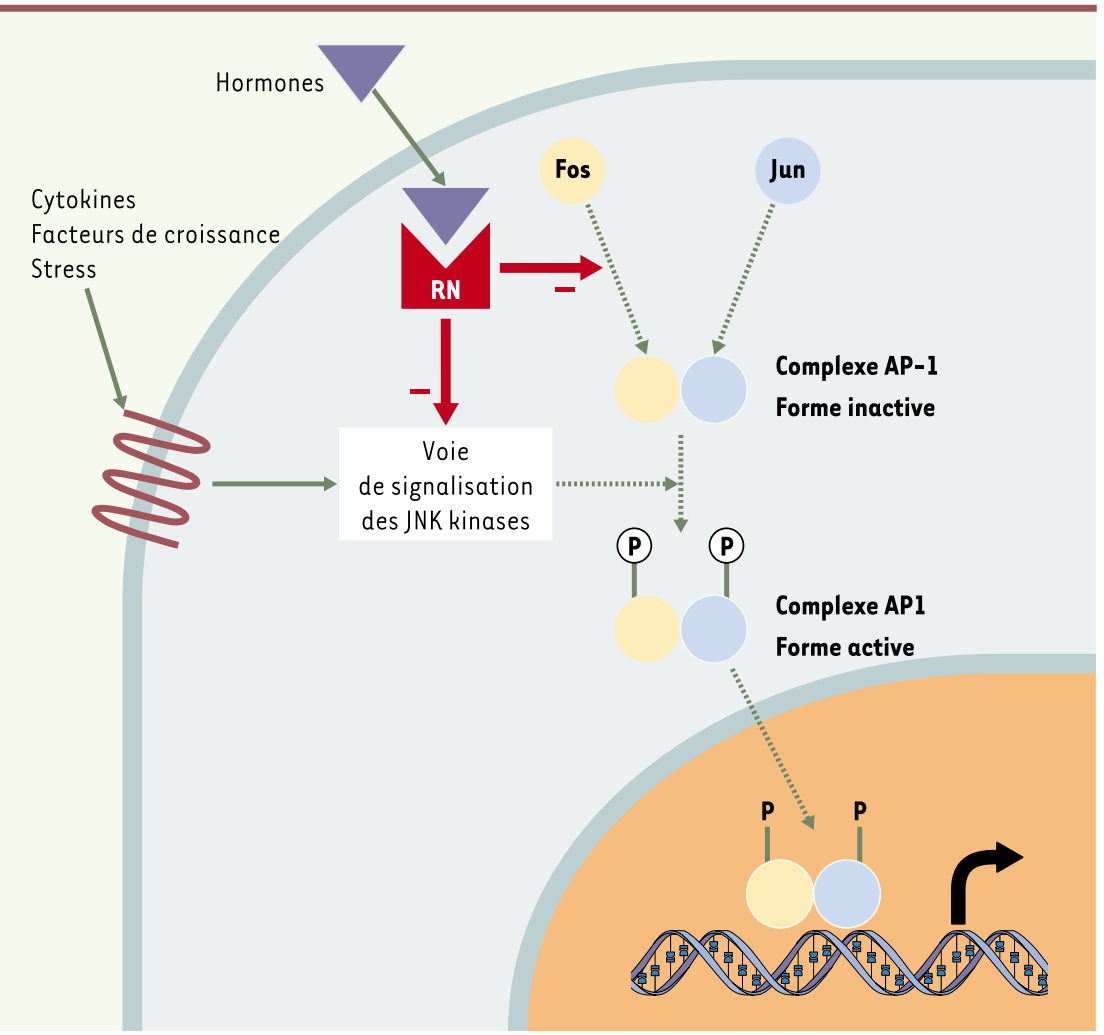

Figure 3. Répression de l'activité transcriptionnelle du complexe AP-I par les récepteurs nucléaires. Les récepteurs nucléaires (RN) peuvent réprimer l'activité transcriptionnelle du complexe AP-l en inhibant sa phosphorylation par la voie des JNK (Jun N-terminal kinases) ou la dimérisation des protéines Jun et Fos [41, 42]. de reconnaissance spécifique d'une séquence nucléotidique mais également un domaine d'interaction protéine/protéine [35]. De fait, tous les récepteurs nucléaires semblent capables d'interagir avec AP-1. Le mécanisme d'antagonisme de type tethering (Figure 2), qui expliquerait les interactions négatives entre le facteur PU.1, membre de la famille ETS, et le facteur GATAl, ne semble pas intervenir pour l'interaction entre récepteurs nucléaires et AP-1 [36-38]. Comme pour l'interaction entre GR et NF- $K B$, on peut évoquer la compétition pour un cofacteur commun présent en quantité limitante et les protéines $S R C-1$ et CBP (CREB binding protein) sont encore une fois des candidats privilégiés. Mais les résultats expérimentaux à ce sujet sont pour l'instant controversés. Deux études ont suggéré que la surexpression de quantités croissantes de SRC- 1 et de CBP permettrait de lever l'inhibition réciproque entre $G R$ et $A P-1[13,39]$ alors qu'un travail plus récent ne montre aucune conséquence de la surexpression des deux cofacteurs [40]. D’autres mécanismes sont invoqués (Figure 3), qui varient selon le partenaire d'AP-1. Le récepteur nucléaire $R A R \alpha$ réprimerait l'activité du complexe AP-1 en inhibant la formation du complexe Jun-Fos (Figure 3) [41] tandis que les récepteurs GR, RAR et TR (thyroid hormone receptor) inhiberaient la cascade de phosphorylation dépendant de JNK (Jun N-terminal kinase), la kinase qui assure la phosphorylation de JUN [21, 40, 42].

\section{Conclusions}

Les interactions négatives entre les récepteurs nucléaires et les facteurs transcriptionnels NF-KB ou AP- 1 sont démonstratifs de la complexité des connexions moléculaires entre les deux grandes voies de signalisation: la voie de différenciation cellulaire relayée par les récepteurs nucléaires et la voie de prolifération cellulaire activée par les autres facteurs transcriptionnels via des récepteurs membranaires. Quel qu'en soit le mécanisme (et il est probable que plusieurs sont nécessaires pour une interaction effective et finement contrôlée), les interactions négatives entre facteurs transcriptionnels ont une grande importance fonctionnelle in vivo. Elles expliquent pour une large part l'activité anti-inflammatoire des glucocorticoïdes ou l'action anti-athéromateuse 
de certains médicaments hypolipémiants comme les fibrates.

Les interférences fonctionnelles exercées entre facteurs transcriptionnels représentent, par leur généralisation à de multiples modèles cellulaires, une fonction biologique majeure de la cellule. Elles semblent communes à toutes les familles de facteurs de transcription et élargissent leurs propriétés fonctionnelles, par exemple en leur permettant de contrôler des gènes cibles sans liaison directe à I'ADN. Elles permettent également une régulation très fine de l'expression des gènes par l'intégration intracellulaire des multiples signaux que la cellule reçoit du milieu extérieur. On peut y voir un moyen pour la cellule de contenir à différents niveaux un débordement éventuel de l'activité transcriptionnelle de certains facteurs connus pour être des oncoprotéines (complexe AP-1, facteurs ETS...). Les points de contrôle exercés par les interactions d'autres facteurs potentiellement oncosupresseurs, comme les récepteurs nucléaires, constituent alors autant de verrous qui doivent être forcés successivement lors des multiples étapes de la carcinogenèse. $\diamond$

\section{SUMMARY}

Negative cross-talk between nuclear receptors and transcription factors: implications in inflammation and oncogenesis

Nuclear receptors are transcription factors mediating a signal pathway that triggers the cell into differentiation. By contrast, membrane receptors mediate a proliferation signal pathway via a phosphorylation cascade that activates transcription factors such as NF-KB and AP- 1 complex. To allow efficient cellular integration of these contradictory signals, transcription factors mutually interact and modulate their transcription activity. Although often synergistic, these interactions can also be negative. They then result from various mechanisms acting either at the transcriptional level (competitive binding to DNA or to a common limitant cofactor...) or upstream DNA binding (inhibition of DNA binding, inhibition of phosphorylation...). Whatever the precise mechanisms, these negative interactions are significant in vivo. For instance, glucocorticoid and PPAR receptors repress the transcription activity of the pro-inflammatory factor NF- $K B$. This partly explains the anti-inflammatory effects of their respective ligands (glucocorticoids and fibrates). Likewise, interactions between nuclear receptors and AP-1 complex are likeky to participate to the anti-oncogenic activity of glucocorticoids and retinoic acid. $\diamond$

\section{RÉFÉRENCES}

1. McKay LI, Cidlowski JA. Molecular control of immune/inflammatory responses: interactions between nuclear factorkappa B and steroid receptor-signaling pathways. Endocrinol Rev 1999; 20: 435-59.

2. McKay LI, Cidlowski JA. Cross-talk between nuclear factor-kappa B and the steroid hormone receptors: mechanisms of mutual antagonism. Mol Endocrinol 1998; 12: 45-56.

3. Auphan N, DiDonato JA, Rosette C, Helmberg A, Karin $M$.

Immunosuppression by glucocorticoids: inhibition of NF-kappa B activity through induction of I kappa B synthesis. Science 1995; 270: 286-90.

4. Scheinman RI, Gualberto $A$, Jewell CM, Cidlowski JA, Baldwin AS Jr.

Characterization of mechanisms involved in transrepression of NFkappa B by activated glucocorticoid receptors. Mol Cell Biol 1995; 15: 943-53.

5. Schouten GJ, van der Eb AJ, Zantema A. Downregulation of MHC class I expression due to interference with p105-NF kappa Bl processing by Adl2ElA. EMBO J 1995; 14 : 1498-507.

6. Heck S, Bender K, Kullmann $M$, et al. I kappaB alphaindependent downregulation of NFkappaB activity by glucocorticoid receptor. EMBO J 1997; 16: 4698-707.

7. Brostjan C, Anrather J, Csizmadia V, et al. Glucocorticoid-mediated repression of NFkappaB activity in endothelial cells does not involve induction of IkappaBalpha synthesis. J Biol Chem 1996 ;

271: 19612-6.
8. De Bosscher K, Vanden Berghe W, Haegeman G. Mechanisms of antiinflammatory action and of immunosuppression by glucocorticoids: negative interference of activated glucocorticoid receptor with transcription factors. J Neuroimmunol 2000; 109: 16-22.

9. Ray A, Prefontaine KE. Physical association and functional antagonism between the $\mathrm{p} 65$ subunit of transcription factor NFkappa $B$ and the glucocorticoid receptor. Proc Natl Acad Sci USA 1994; 91: 752-6.

10. Baes M, Castelein H, Desmet L, Declercq PE. Antagonism of COUP-TF and PPAR alpha/RXR alpha on the activation of the malic enzyme gene promoter: modulation by 9 -cis RA. Biochem Biophys Res Commun 1995; 215: 338-45.

11. Ikeda $Y$, Sugawara $A$, Taniyama $Y$, et al. Suppression of rat thromboxane synthase gene transcription by peroxisome proliferatoractivated receptor gamma in macrophages via an interaction with NRF2. J Biol Chem 2000; 275: 33142-50.

12. Sheppard KA, Phelps KM, Williams AJ, et al. Nuclear integration of glucocorticoid receptor and nuclear factor-kappaB signaling by CREB-binding protein and steroid receptor coactivator-1. J Biol Chem 1998; 273: 29291-4.

13. Kamei $Y, X_{u} L$, Heinzel T, et al. A CBP integrator complex mediates transcriptional activation and AP- 1 inhibition by nuclear receptors. Cell 1996; 85: 403-14. 
14. McKay LI, Cidlowski JA. CBP (CREB binding protein) integrates NF-kappaB (nuclear factor-kappaB) and glucocorticoid receptor physical interactions and antagonism. Mol Endocrinol 2000; 14: 1222-34.

15. Webster JC, Oakley RH, Jewell CM, Cidlowski JA. Proinflammatory cytokines regulate human glucocorticoid receptor gene expression and lead to the accumulation of the dominant negative beta isoform: a mechanism for the generation of glucocorticoid resistance. Proc Natl Acad Sci USA 2001; 98: 6865-70.

16. Supakar $P C$, Jung $M H$, Song CS, Chatterjee B, Roy AK. Nuclear factor kappa B functions as a negative regulator for the rat androgen receptor gene and NF-kappa B activity increases during the agedependent desensitization of the liver. J Biol Chem 1995; 270: 837-42.

17. Kalkhoven $\varepsilon$, Wissink $S$, van der Saag PT, van der Burg B. Negative interaction between the RelA(p65) subunit of NF-kappaB and the progesterone receptor. J Biol Chem 1996; 271: 6217-24.

18. Ray P, Ghosh SK, Zhang DH, Ray A. Repression of interleukin- 6 gene expression by 17 betaestradiol: inhibition of the DNA-binding activity of the transcription factors NF-IL6 and NF-kappa B by the estrogen receptor. FEBS Lett 1997; 409: 79-85.

19. Stein B, Yang MX. Repression of the interleukin- 6 promoter by estrogen receptor is mediated by NF-kappa B and $C / \varepsilon B P$ beta. Mol Cell Biol 1995; 15: 4971-9.

20. Staels B, Koenig W, Habib $A$, et al. Activation of human aortic smoothmuscle cells is inhibited by PPARalpha but not by PPARgamma activators. Nature 1998; 393: 790-3.
21. Delerive $P$, De Bosscher $K$, Besnard S, et al. Peroxisome proliferatoractivated receptor alpha negatively regulates the vascular inflammatory gene response by negative cross-talk with transcription factors NFkappaB and AP-1. $J$ Biol Chem 1999; 274: 32048-54.

22. Hansen LA, Sigman CC, Andreola F, et al. Retinoids in chemoprevention and differentiation therapy. Carcinogenesis 2000; 21: 1271-9.

23. Darby TG, Meissner JD, Ruhlmann A, Mueller WH, Scheibe RJ. Functional interference between retinoic acid or steroid hormone receptors and the oncoprotein Fli-1. Oncogene 1997; 15: 3067-82.

24. Ben-David Y, Bernstein A. Friend virus-induced erythroleukemia and the multistage nature of cancer. Cell 1991; 66: 831-4.

25. Diamond MI, Miner JN, Yoshinaga SK, Yamamoto KR. Transcription factor interactions: selectors of positive or negative regulation from a single DNA element. Science 1990; 249: 1266-72.

26. Schule R, Rangarajan $P$, Yang $N$, et al. Retinoic acid is a negative regulator of AP-1-responsive genes. Proc Natl Acad Sci USA 1991; 88: 6092-6.

27. Schule R, Evans RM. Crosscoupling of signal transduction pathways: zinc finger meets leucine zipper. Trends Genet 1991; 7: 377-81.

28. Jonat C, Rahmsdorf HJ, Park KK, et al. Antitumor promotion and antiinflammation: downmodulation of AP-1 (Fos/Jun) activity by glucocorticoid hormone. Cell 1990; 62: 1189-204.
29. Yang-Yen HF, Chambard JC, Sun $Y L$, et al.

Transcriptional interference between c-Jun and the glucocorticoid receptor: mutual inhibition of DNA binding due to direct protein-protein interaction. Cell 1990; 2: 1205-15.

30. Nicholson RC, Mader S, Nagpal S, et al. Negative regulation of the rat stromelysin gene promoter by retinoic acid is mediated by an API binding site. EMBO J 1990; 9: 4443-54.

31. Zhang XK, Wills KN, Husmann M, Hermann T, Pfahl M. Novel pathway for thyroid hormone receptor action through interaction with jun and fos oncogene activities. Mol Cell Biol 1991; 11: 6016-25.

32. Doucas V, Spyrou G, Yaniv M. Unregulated expression of c-Jun or c-Fos proteins but not Jun $D$ inhibits oestrogen receptor activity in human breast cancer derived cells. EMBO J 1991; 10: 2237-45.

33. Schule R, Umesono $K$, Mangelsdorf DJ, et al. JunFos and receptors for vitamins $A$ and $D$ recognize a common response element in the human osteocalcin gene. Cell 1990; 61: 497-504.

34. Lucibello FC, Slater EP, Jooss KU, Beato M, Muller R. Mutual transrepression of Fos and the glucocorticoid receptor: involvement of a functional domain in Fos which is absent in FosB. EMBO J 1990; 9: 2827-34.

35. Wagner $S$, Green MR. DNAbinding domains : targets for viral and cellular regulators. Curr Opin Cell Biol 1994; 6: 410-4.
36. Rekhtman N, Radparvar F, Evans T, Skoultchi Al. Direct interaction of hematopoietic transcription factors PU.1 and GATA- 1 : functional antagonism in erythroid cells. Genes Dev 1999; 13: 1398-411.

37. Zhang P, Behre G, Pan J, et al. Negative cross-talk between hematopoietic regulators: GATA proteins repress PU.1. Proc Natl Acad Sci USA 1999, 96: 8705-10.

38. Nerlov C, Querfurth $\varepsilon$, Kulessa H, Graf T. GATA-1 interacts with the myeloid PU.l transcription factor and represses PU.1dependent transcription. Blood 2000; 95: 2543-51.

39. Lee SK, Kim HJ, Na Sy, et al. Steroid receptor coactivator- 1 coactivates activating protein-1mediated transactivations through interaction with the c-Jun and c-Fos subunits. J Biol Chem 1998; 273: 16651-4.

40. De Bosscher K, Vanden Berghe W, Haegeman $G$. Glucocorticoid repression of AP-1 is not mediated by competition for nuclear coactivators. Mol Endocrinol 2001. 15: 219-27.

41. Zhou XF, Shen XQ, Shemshedini L. Ligandactivated retinoic acid receptor inhibits AP-1 transactivation by disrupting c-Jun/c-Fos dimerization. Mol Endocrinol 1999; 13: 276-85.

42. Caelles C, Gonzalez-Sancho JM, Munoz A. Nuclear hormone receptor antagonism with AP-1 by inhibition of the JNK pathway. Genes Dev 1997; 11: 3351-64.
TIRÉS À PART

V. Vlaeminck-Guillem 